Article

\title{
Evolution of Hemodynamic Parameters Simulated by Means of Diffusion Models
}

\author{
Andrzej Walczak ${ }^{1, *}$, Paweł Moszczyński ${ }^{1}$ and Paweł Krzesiński ${ }^{2}$ (D) \\ 1 Institute of Computer and Information Systems, Military University of Technology, ul. gen. Kaliskiego 2, \\ 00-908 Warsaw, Poland; pawel.moszczynski@wat.edu.pl \\ 2 Department of Cardiology and Internal Diseases, Military Institute of Medicine, ul. Szaserów 128, \\ 04-141 Warsaw, Poland; pkrzesinski@wim.mil.pl \\ * Correspondence: andrzej.walczak@wat.edu.pl
}

check for updates

Citation: Walczak, A.; Moszczyński, P.; Krzesiński, P. Evolution of Hemodynamic Parameters Simulated by Means of Diffusion Models. Appl. Sci. 2021, 11, 11412. https://doi.org/ 10.3390/app112311412

Academic Editors: Kiril Tenekedjiev, Atsushi Mase and Mike Calford

Received: 28 September 2021

Accepted: 29 November 2021

Published: 2 December 2021

Publisher's Note: MDPI stays neutra with regard to jurisdictional claims in published maps and institutional affiliations.

Copyright: (c) 2021 by the authors. Licensee MDPI, Basel, Switzerland. This article is an open access article distributed under the terms and conditions of the Creative Commons Attribution (CC BY) license (https:// creativecommons.org/licenses/by/ $4.0 /)$.

\begin{abstract}
Diffusion is a well-known physical phenomenon governing such processes as movement of particles or transportation of heat. In this paper, we prove that a close analogy to those processes exists in medical data behavior, and that changes in the values of medical parameters measured while treating patients may be described using diffusion models as well. The medical condition of a patient is usually described by a set of discrete values. The evolution of that condition and, consequently, of the disease has the form of a transition of that set of discrete values, which correspond to specific parameters. This is a typical medical diagnosis scheme. However, disease evolution is a phenomenon that is characterized by continuously varying, temporal characteristics. A mathematical disease evolution model is, in fact, a continuous diffusion process from one discrete slot of the diagnosed parameter value to another inside the mentioned set. The ability to predict such diffusion-related properties offer precious support in diagnostic decision-making. We have examined several hundred patients while conducting a medical research project. All patients were under treatment to stabilize their hemodynamic parameters. A diffusion model relied upon simulating the results of treatment is proposed here. Time evolution of thoraric fluid content (TFC) has been used as the illustrative example. The objective is to prove that diffusion models are a proper and convenient solution for predicting disease evolution processes. We applied the Fokker-Planck equation (FPE), considering it to be most adequate for examining the treatment results by means of diffusion. We confirmed that the phenomenon of diffusion explains the evolution of the heart disease parameters observed. The evolution of TFC has been chosen as an example of a hemodynamic parameter.
\end{abstract}

Keywords: Fokker-Planck equation; diffusion; heart failure; impedance cardiography; thoracic fluid content

\section{Introduction}

The current state of the art in predicting disease evolution may be summarized in the following manner. Hemodynamic assessment procedures are widely described in the literature, primarily by means of biological models for flow or response functions [1-4]. Numerous investigations aimed to estimate the risk of heart failure by relying on common statistical data processing tools and regression models [5-7]. Highly illustrative investigations were proposed by Lassnig at al. [8]. Other clinical prediction models (CPMs), also known as clinical prediction scores or rules, are used to estimate the risk of an existing disease (diagnostic prediction model) or future outcomes (prognostic prediction model) for a given individual and consist of analyzing the values of numerous predictors (prognostic or risk factors), such as age, sex, and biomarkers [9-12]. Generic prediction models are widely used in adult intensive care medicine. These include, for instance, acute physiology and chronic health evaluation (APACHE) II, APACHE III, APACHE IV, simplified acute physiology score (SAPS) II, SAPS 3, and mortality probability model III [13]. So, different 
predictive approaches are present in the literature. The results presented in there are organized in accordance with risk analysis, and such an approach does not allow us to simulate disease evolution. The following question arises: are we able to simulate the evolution of hemodynamic parameters during medical treatment? In this paper, we propose a model explaining the rules of hemodynamic parameter evolution in patients with heart failure. Simulation of the disease evolution seems to be possible with the presented approach.

The activities undertaken may be divided into experimental and theoretical phases. In the experimental phase of the project, a multicenter, prospective, randomized, openlabel, and controlled, parallel group trial was conducted (ClinicalTrials.gov Identifier: NCT03476590). Here, 605 patients suffering from heart failure were recruited to participate in the project. In the theoretical phase, we propose to describe the behavior of the hemodynamic parameters measured by relying on diffusion models.

In Section 2, experimental observations and analyses are presented. Section 3 presents theoretical models describing the diffusion phenomena. Section 3 contains the results of confrontation theoretical models with experimental data. Finally, Section 4 contains a summary and a discussion.

The achieved aim is the creation of a continuous, differential model for disease evolution.

\section{Experimental Data}

The experimental data used in this study were sourced from the AMULET research project [14], under which a multicenter, prospective, randomized, open-label and controlled, parallel group trial was conducted (ClinicalTrials.gov Identifier: NCT03476590) at nine locations throughout Poland. In total, 605 patients with heart failure were recruited. To our study, we examined electrocardiogram (ECG—electrocardiogram) and impedance (ICGimpedance cardiogram) curves recorded with the use of an ICG device (Cardioscreen 2000, Medis, Illmenau, Germany). This non-invasive diagnostic method allows one to collect a set of specific hemodynamic parameters, such as: heart rate (HR-heart rate), diastolic and systolic blood pressure (DBP — diastolic blood pressure, $\mathrm{SBP}$ - systolic blood pressure), stroke volume (SV-stroke volume), and thoracic fluid content (TFC—-thoraric fluid content). In our analysis, TFC has been used as an illustrative example. The value of TFC is the inverse of chest impedance with unit (1/Ohm).

TFC values were measured during a clinical examination of 605 patients, performed in a relaxed, seated position [14]. The total number of measurements made is 2860 , with the number of individual patient observations differing for each patient within the measurement set. The results registered are illustrated in Figure 1. At least half of the patients were investigated several times, with the observation period lasting for up to 12 months per patient.

All intervals of the investigated TFC value have been divided arbitrarily into 15 discrete value slots, as shown in Figure 1. The assumed number of slots must be greater than five to allow statistical analysis (especially $\chi^{2}$ tests) but not too high to avoid complexity of calculations. With computer calculation, we estimate that 15 slots allow an acceptable accuracy of calculations. The time series was registered as follows: due to the irregular flow of the measured data, we adopted a registration period that was 31 days long for each slot. The result of the measurement was placed inside a given slot if number of days between the sequenced examination of the patient remained within the $\mathrm{k} \pm 0.5$ range, with $\mathrm{k}$ being the duration of the registration period between measurements. Each slot includes hits counted during measurements registered over a five-month period. Hits registered during each single measurement period are illustrated below.

One can observe that, for the increasing $\Delta t$, the total number of TFC hits registered tends to have the same stationary distribution of TFC, as shown in Figure 1. We assumed that the period of 5 months during which the measurements were made (see Figure 2 and comments to Figure 1) is sufficient for proper estimation of stationary distribution. 


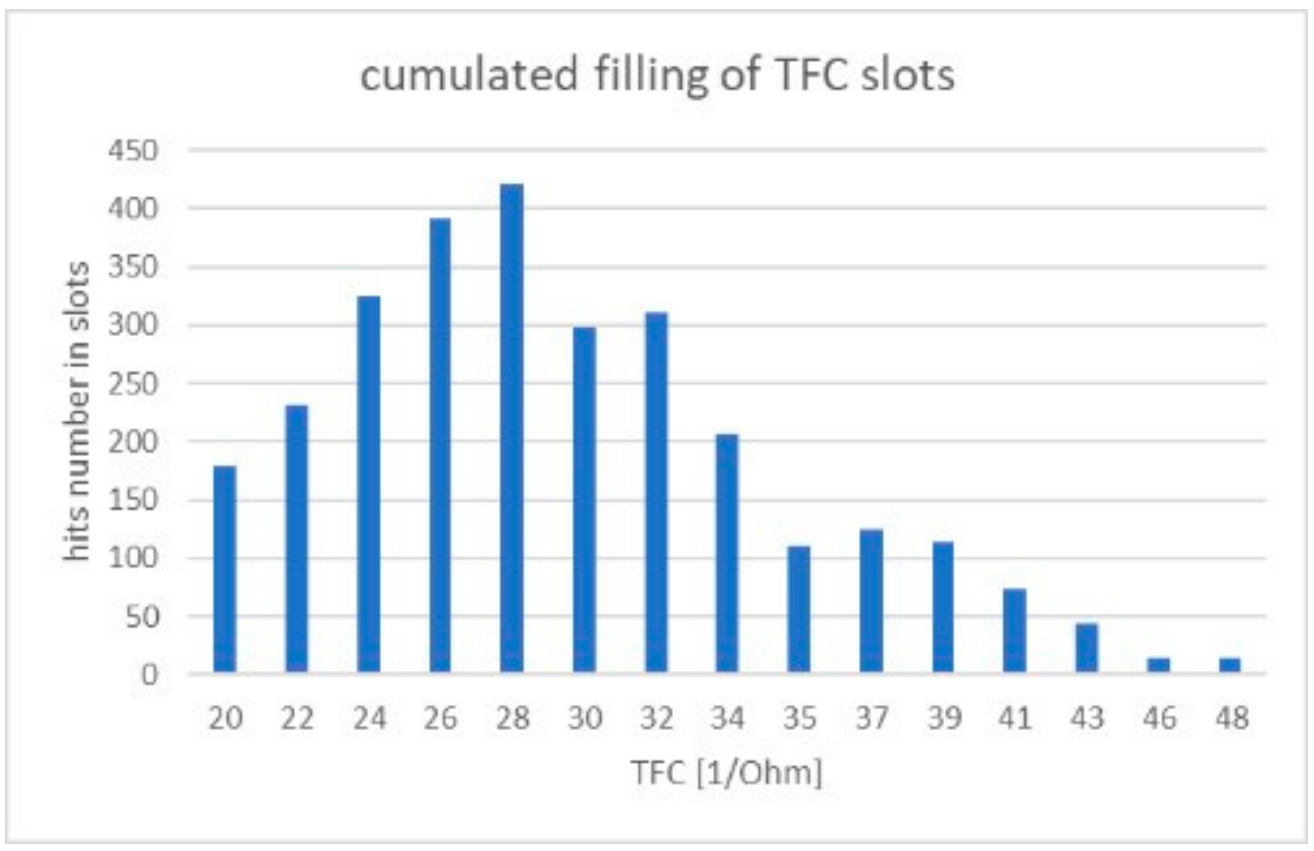

Figure 1. Values of TFC slots filled with the number of measurements.

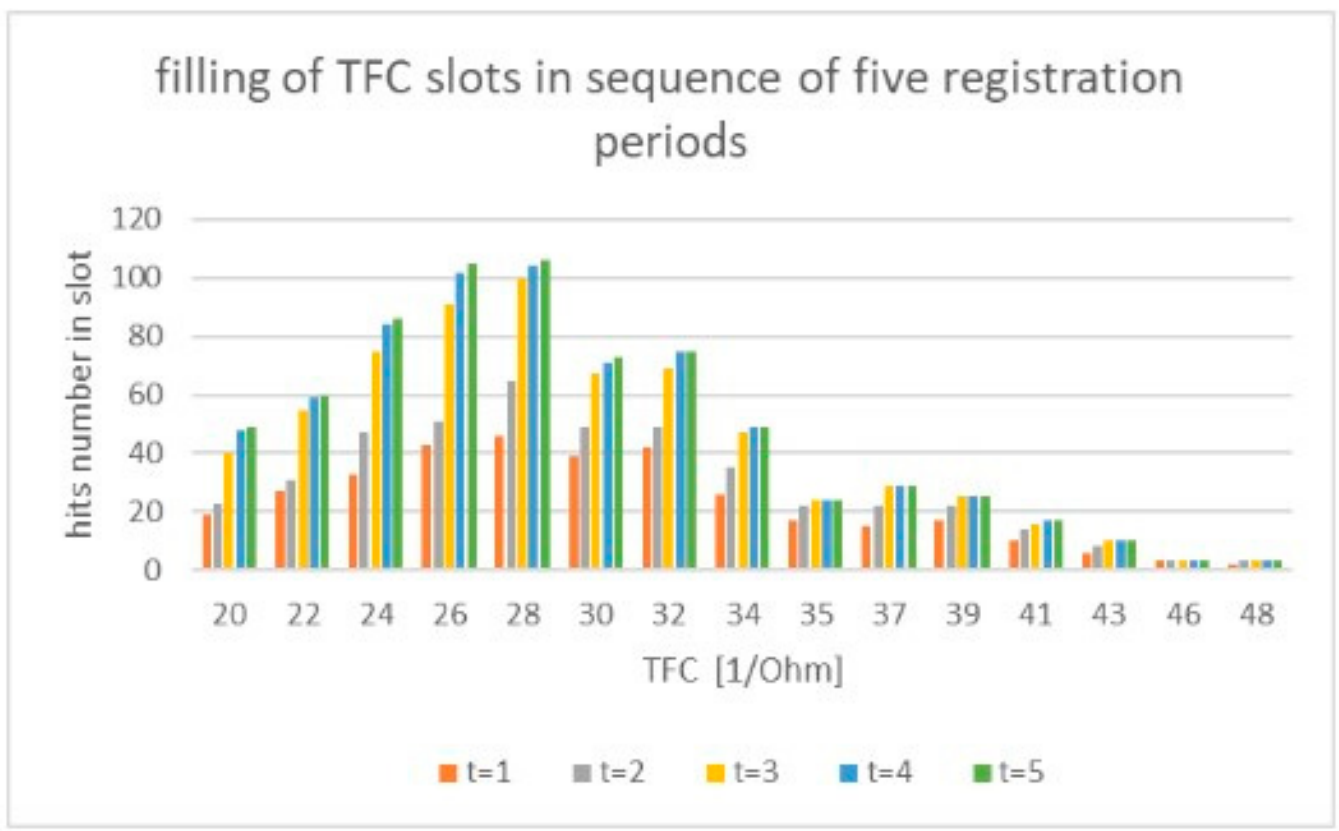

Figure 2. Stepped registration of TFC values in measurement intervals denoted as $t$ in the figure.

\section{Theoretical Model and Calculation}

The Fokker-Planck equation with one variable (here, variable $x$ denotes the TFC value) has the following form [15]:

$$
\frac{\partial P}{\partial t}=\left[-\frac{\partial}{\partial x} D^{(1)}(x)+\frac{\partial^{2}}{\partial x^{2}} D^{(2)}(x)\right] P
$$

In this equation, $D^{(2)}(x)>1$ is the diffusion coefficient, and $D^{(1)}(x)$ is called the drift coefficient. In general, both coefficients may also depend on time. The equation describes 
behavior $P(x, t)$, i.e., the $P(x, t)$ is distribution of probability. We assumed stochastic process characterizes the linear drift coefficient, and the diffusion coefficient is constant.

$$
\frac{\partial P}{\partial t}=\gamma \frac{\partial(x P)}{\partial x}+D \frac{\partial^{2}}{\partial x^{2}} P
$$

In our experiment, drift $\gamma x$ is present due to therapy data registration, so drift presence is necessary. Diffusion describes the transition between slots of parameter values measured. A solution of Equation (2) is of the form [15]:

$$
P\left(x, t \mid x^{\prime}, t^{\prime}\right)=\sqrt{\frac{\gamma}{2 \pi D\left(1-e^{-2 \gamma\left(t-t^{\prime}\right)}\right)}} \exp \left(-\frac{\gamma\left(x-x^{\prime} e^{-\gamma\left(t-t^{\prime}\right)}\right)^{2}}{2 D\left(1-e^{-2 \gamma\left(t-t^{\prime}\right)}\right)}\right)
$$

Green function (3) of Equation (2) is one of the basic ways for system dynamic description $[15,16]$. The stationary solution for $\gamma>0$ and a sufficiently long-period $\gamma\left(t-t^{\prime}\right)>>1$ takes the following form:

$$
W(x)=\sqrt{\frac{\gamma}{2 \pi D}} \exp \left(\frac{-\gamma x^{2}}{2 D}\right)
$$

For $\gamma \leq 0$, no stationary solutions exist. Formal mathematical procedures enable us to find coefficients $\gamma$ and $D$ with a normalization condition and a boundary condition. Due to the existence of a stationary shape of $W(x)$ distribution obtained in the experiment, we can assume that the boundary conditions are properly fulfilled despite not being fully established in the formal way. In such a situation, the assumption that coefficients $\gamma$ and $D$ are well-matched by means of conjugated gradient algorithm and, simultaneously, to minimize root mean square error (RMSE) between the experiment data and the theoretical model (4) is permissible.

The set of solutions for $W(x)$ is placed in Table 1, where local and global minima have been shown for the illustrative subset of $\gamma$ and $D$. The shape for stationary distribution with $\gamma$ and $D$ for the global minimum is presented in Figure 3 .

Table 1. Illustrative values of RMSE, with the global minimum highlighted in bold print obtained by means of conjugated gradient algorithm.

\begin{tabular}{ccc}
\hline RMSE & $\gamma$ & D \\
\hline 0.09810952 & 0.4 & 11.99 \\
\hline 0.09838436 & 0.06 & 1.75 \\
\hline 0.09811257 & 0.2 & 5.99 \\
\hline 0.09811050 & 0.3 & 8.99 \\
\hline
\end{tabular}

The results obtained with the conjugated gradient solution are placed in Table 1. The global minimum is placed in the first row of Table 1.

The green function of FPE with adjusted $\gamma$ and $D$ values, and stationary distribution $W\left(x^{\prime}\right)$ is applied to determine $P(x, t)$ distribution in accordance with:

$$
P(x, t)=\int P\left(x, t \mid x^{\prime}-x_{0}\right) W\left(x^{\prime}-x_{0}\right) d x^{\prime}
$$

Distribution $P(x, t)$ allows us to verify if the calculated and measured evolution of TFC values is statistically convergent by conducting an $\chi^{2}$ test. Item $x_{0}$ is commonly the maximum observed value of $W\left(x^{\prime}\right)$ or may also be the boundary point. A comparison of the theoretical $P(x, t)$ with the experiment's results is presented in Table 2 , and the outcomes of statistical tests are shown in Table 3. 


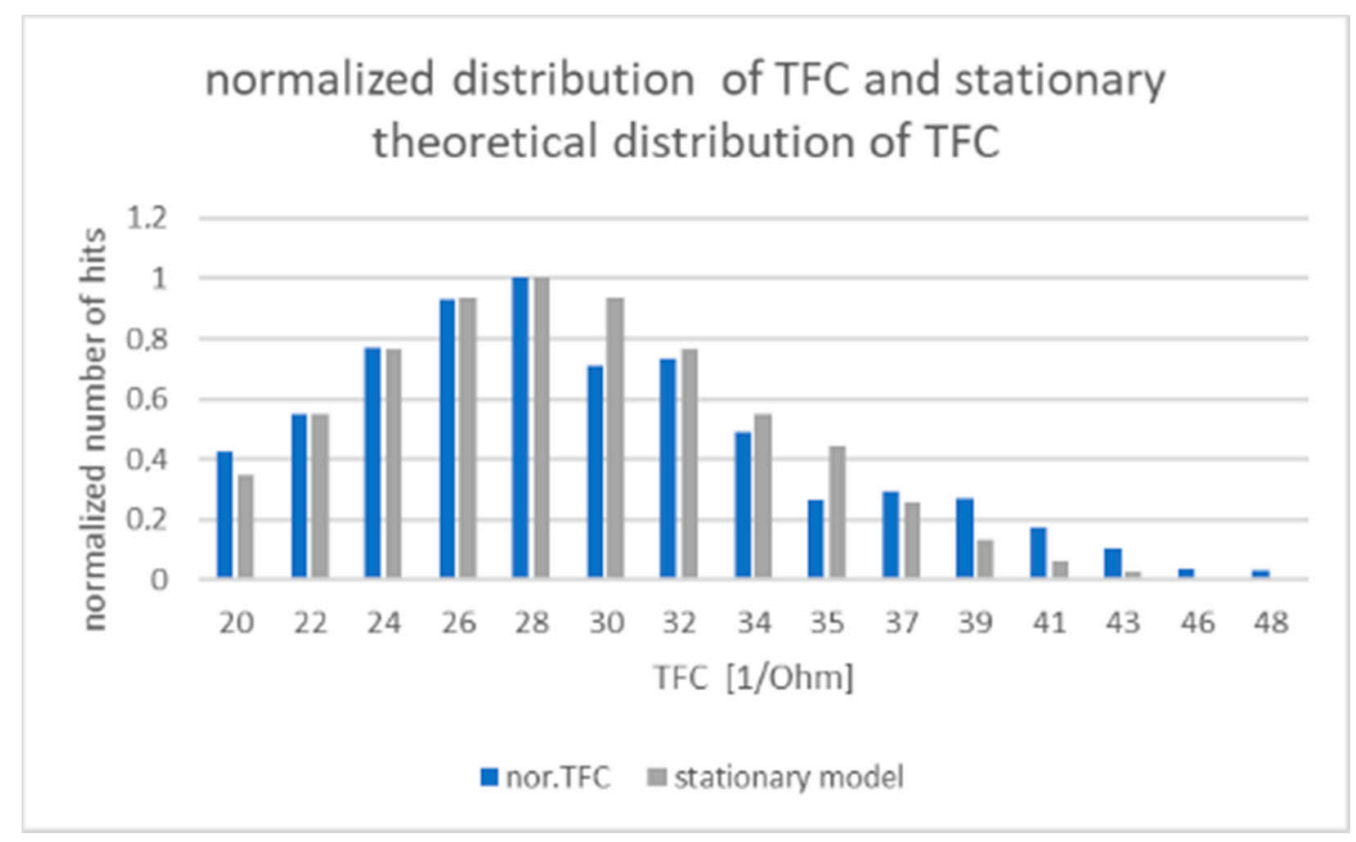

Figure 3. Result of minimization of RMSE between experimental TFC distribution data and the stationary distribution model $W(x)$.

Table 2. The TFC slots filling obtained during measurements (see also Figure 2 ) and values predicted with $P(x, t)$.

\begin{tabular}{|c|c|c|c|c|c|c|c|c|}
\hline \multirow[t]{2}{*}{$\begin{array}{l}\text { TFC Slot } \\
\text { Number }\end{array}$} & \multicolumn{2}{|c|}{$\begin{array}{c}\text { Observation Period } \\
\text { t2-t } 1\end{array}$} & \multicolumn{2}{|c|}{$\begin{array}{c}\text { Observation Period } \\
\mathrm{t} 3-\mathrm{t} 2\end{array}$} & \multicolumn{2}{|c|}{$\begin{array}{c}\text { Observation Period } \\
\mathrm{t} 4-\mathrm{t} 3\end{array}$} & \multicolumn{2}{|c|}{$\begin{array}{c}\text { Observation Period } \\
\text { t5-t4 }\end{array}$} \\
\hline & $\begin{array}{l}\text { Registered } \\
\text { hits of TFC }\end{array}$ & $\begin{array}{l}\text { Predicted } \\
\text { TFC hits }\end{array}$ & $\begin{array}{l}\text { Registered } \\
\text { hits of TFC }\end{array}$ & $\begin{array}{l}\text { Predicted } \\
\text { TFC hits }\end{array}$ & $\begin{array}{l}\text { Registered } \\
\text { hits of TFC }\end{array}$ & $\begin{array}{l}\text { Predicted } \\
\text { TFC hits }\end{array}$ & $\begin{array}{l}\text { Registered } \\
\text { hits of TFC }\end{array}$ & $\begin{array}{l}\text { Predicted } \\
\text { TFC hits }\end{array}$ \\
\hline 1 & 19 & 18.9475 & 23 & 22.8236 & 40 & 29.9024 & 48 & 28.6218 \\
\hline 2 & 27 & 31.5513 & 31 & 39.8768 & 55 & 55.6347 & 59 & 55.9634 \\
\hline 3 & 33 & 40.8633 & 47 & 53.8865 & 75 & 79.7512 & 84 & 84.2399 \\
\hline 4 & 43 & 39.6876 & 51 & 55.9473 & 91 & 87.9519 & 102 & 97.5805 \\
\hline 5 & 46 & 37.5247 & 65 & 54.1467 & 100 & 86.493 & 104 & 97.1694 \\
\hline 6 & 39 & 39.6876 & 49 & 55.7919 & 67 & 83.7667 & 71 & 89.5681 \\
\hline 7 & 42 & 40.8643 & 49 & 44.0522 & 69 & 62.3851 & 75 & 63.5276 \\
\hline 8 & 26 & 31.5513 & 35 & 15.541 & 47 & 20.981 & 49 & 20.549 \\
\hline 9 & 17 & 10.9594 & 22 & 19.0808 & 24 & 24.5837 & 24 & 23.2358 \\
\hline 10 & 15 & 13.509 & 22 & 7.9594 & 29 & 9.5469 & 29 & 8.5716 \\
\hline 11 & 17 & 5.8755 & 22 & 2.5746 & 25 & 2.8579 & 25 & 2.4346 \\
\hline 12 & 10 & 0.7917 & 14 & 0.7780 & 16 & 0.6587 & 17 & 0.5322 \\
\hline 13 & 6 & 0.5562 & 8 & 0.6613 & 10 & 0.1285 & 10 & 0.0982 \\
\hline 14 & 3 & 0.1235 & 3 & 0 & 3 & 0 & 3 & 0 \\
\hline
\end{tabular}


Table 3. Calculations of $\chi^{2}$ test for sequence of period of measurement.

\begin{tabular}{cccc}
\hline $\begin{array}{c}\text { Time Period in } \\
\text { Experiment }\end{array}$ & $\chi^{2}$ Test Value & $\begin{array}{c}\text { Number of Independent } \\
\text { Variables }\end{array}$ & $\begin{array}{c}\text { Significance Level } \\
\text { (Critical Value } \boldsymbol{\alpha})\end{array}$ \\
\hline $\mathrm{t} 2-\mathrm{t} 1$ & 314,375 & 14 & $\alpha=0.005$ \\
\hline $\mathrm{t} 3-\mathrm{t} 2$ & 570,858 & 14 & $\alpha<0.001$ \\
\hline $\mathrm{t} 4-\mathrm{t} 3$ & 811,461 & 14 & $\alpha<0.001$ \\
\hline $\mathrm{t} 5-\mathrm{t} 4$ & 922,901 & 14 & $\alpha<0.001$ \\
\hline
\end{tabular}

\section{Discussion and Conclusions}

Claiming that hemodynamic parameter values measured in a population of patients behave in accordance with the rules of drifted diffusion, we have identified a step-by-step procedure allowing to verify such a statement. From the green function of FPE, we obtained stationary distribution $W(x)$ of the thoracic fluid content (TFC) parameter observed, and by minimizing RMSE between the theoretical model and the experimental observations of stationary distribution, we adjusted the parameters of the stochastic process with the use of conjugated gradient algorithms. It turned out that the resulting form of the stochastic process is the Ornstein-Uhlenbeck process. So, we found the Ornstein-Uhlenbeck diffusion process as a model for medical treatment of heart failures. From the analytical form of the FPE solution (green function of FPE), we determined the "ex definition" distribution $P(x, t)$ for the observed hemodynamic parameters and determined dynamics of TFC evolution. The last step had the form of a statistical $\chi^{2}$ test, aiming to accept or to reject the proposed theoretical model. The $\chi^{2}$ test confirms the accepted level of efficiency of the theoretical model of the Ornstein-Uhlenbeck process, describing TFC evolution.

Finally, we may conclude that the diffusion processes have turned out to be useful tools for predicting disease evolution. We have also proved that:

1. The Ornstein-Uhlenbeck process, including the linear drift component, seems to be a precise tool for simulating the evolution of TFC and, supposedly, other hemodynamic parameters, also during medical treatment.

2. We obtained the distribution $P(x, t)$, allowing us to predict and simulate TFC evolution.

3. Parameter $\gamma$ describes the effectiveness of medical treatment in the population of patients, thus being a mathematical measure of such treatment effectiveness that is worth being analyzed in a more thorough manner, e.g., by focusing on variations depending on sex, occupation, age, severity of heart failure in accordance with the NYHA standard, etc.

The idea of diffusion occurring inside a potential force field is deeply exploited in solid state physics and quantum electronics [16-22], but its successful application in medical diagnostics seems to be a new approach that suggests, to some extent, the existence of a "hidden general symmetry" between physics and biology as well. Diffusion theory is relevant not only for microscopic observations made in physics, but also for much larger scale phenomena, e.g., in medicine. In medicine, fluctuations take place due to multiple and multilevel interactions occurring in the patient's system. Such fluctuations are hard to describe in a precise manner, although their presence seems to be obvious.

The results presented herein are based on data processed during a medical experiment and, to the best of the authors' knowledge, form the first model of its type proposed in medical diagnostics. The results we obtained allows us to predict the time evolution of TFC. Simultaneously, we have confirmed that the fluctuation theory, reflected here by means of $\mathrm{FPE}$, is an inherent element of medical diagnostic processes.

It must be underlined that prospective validation of the model is continued now in collaborative clinics under Amulet project. We do suppose that limitations of the model will be analyzed during further validation. An evident strength of the model is its continuous, differential model of disease evolution. As far as authors know, it seems to be a new approach. 
Author Contributions: Conceptualization, methodology, software, formal analysis-A.W.; validation and statistics-P.M.; investigation, resources-P.K. All authors have read and agreed to the published version of the manuscript.

Funding: This work was supported by the National Centre for Research and Development under the program 'Prevention and treatment of lifestyle diseases'-STRATEGMED III STRATEGMED3/305274/ 8/NCBR/2017).

Institutional Review Board Statement: The study was conducted according to the guidelines of the Declaration of Helsinki, and approved by the Institutional Ethics Committee of Military Institute of Medicine (No. 70/WIM/2016).

Informed Consent Statement: Informed consent was obtained from all subjects involved in the study.

Data Availability Statement: The data used to support the findings of this study are available from the corresponding author upon request.

Acknowledgments: The authors would like to thank the medical staff of their centers for the nursing care and data collection.

Conflicts of Interest: The authors declare that there are no conflict of interest regarding the publication of this paper.

\section{References}

1. Chen, X.; Schwarz, K.Q.; Phillips, D.; Steinmetz, S.D.; Schlief, R. A Mathematical Model for the Assessment of Hemodynamic Parameters Using Quantitative Contrast Echocardiography. IEEE Trans. Biomed. Eng. 1998, 45, 754-765. [CrossRef] [PubMed]

2. Aquinoab, K.M.; Robinsonab, P.A.; Drysdaleab, P.M. Spatiotemporal hemodynamic response functions derived from physiology. J. Theor. Biol. 2014, 347, 118-136. [CrossRef] [PubMed]

3. Mukkamala, R.; Gao, M. A Comparative Analysis of Reduced Arterial Models for Hemodynamic Monitoring. In Proceedings of the 2013 35th Annual International Conference of the IEEE Engineering in Medicine and Biology Society (EMBC), Osaka, Japan, 3-7 July 2013; pp. 225-228. [CrossRef]

4. Morris, P.; Ryan, D.; Morton, A.; Lycett, R.; Lawford, P.; Hose, D.R.; Gunn, J. Virtual Fractional Flow Reserve from Coronary Angiography: Modeling the significance of coronary lesions. JACC Cardiovasc. Interv. 2013, 6, 149-157. [CrossRef] [PubMed]

5. Priyanka, H.; Vivek, R. Multi Model Data Mining Approach for Heart Failure Prediction. Int. J. Data Min. Knowl. Manag. Process 2016, 6, 31-37.

6. Bouvy, M.L.; Heerdink, E.R.; Leufkens, H.G.M.; Hoes, A.W. Predicting mortality in patients with heart failure: A pragmatic approach. Heart 2003, 89, 605-609. [CrossRef] [PubMed]

7. Jiang, W.; Siddiqui, S.; Barnes, S.; Barouch, L.A.; Korley, F.; Martinez, D.; Toerper, M.; Cabral, S.; Hamrock, E.; Levin, S. Readmission Risk Trajectories for Patients with Heart Failure Using a Dynamic Prediction Approach: Retrospective Study. JMIR Med. Inf. 2019, 7, e14756. [CrossRef] [PubMed]

8. Lassnig, A.; Rienmueller, T.; Kramer, D.; Leodolter, W.; Baumgartner, C.; Schroettner, J. A novel hybrid modeling approach for the evaluation of integrated care and economic outcome in heart failure treatment. BMC Med. Inform. Decis. Mak. 2019, 19, 229. [CrossRef] [PubMed]

9. Steyerberg, E.; Moons, K.; Van der Windt, D.; Hayden, J.; Perel, P.; Schroter, S.; Riley, R.D.; Hemingway, H.; Altman, D.G.; PROGRESS Group. Prognosis Research Strategy (PROGRESS) 3: Prognostic Model Research. PLoS Med. 2013, 10, e1001381. [CrossRef] [PubMed]

10. Labarère, J.; Bertran, R.; Fine, M.J. How to derive and validate clinical prediction models for use in intensive care medicine. Intens. Care Med. 2014, 40, 513-527. [CrossRef] [PubMed]

11. Laupacis, N.; Sekar, N.; Stiell, I. Clinical prediction rules. A review and suggested modifications of methodological standards. JAMA 1997, 277, 488-494. [CrossRef] [PubMed]

12. Moons, K.; Altman, D.; Vergouwe, Y.; Royston, P. Prognosis, and prognostic research: Application and impact of prognostic models in clinical practice. BMJ 2009, 338, b606. [CrossRef] [PubMed]

13. Vincent, J.; Moreno, R. Clinical review: Scoring systems in the critically ill. Crit. Care 2010, 14, 207. [CrossRef] [PubMed]

14. Krzesiński, P.; Siebert, J.; Jankowska, A.E.; Galas, A.; Piotrowicz, K.; Stańczyk, A.; Siwołowski, P.; Gutknecht, P.; Chrom, P.; Murawski, P.; et al. Nurse-led ambulatory care supported by non-invasive haemodynamic assessment improves the functional state and well-being of patients suffering from acute heart failure decompensation-A pilot study. ESC Heart Fail. 2021, 8 , 1018-1026. [CrossRef] [PubMed]

15. Risken, H. The Fokker-Planck equation. In Method of Solution and Applications; Springer: Berlin/Heidelberg, Germany, 1989.

16. Gardiner, C.W. Handbook of Stochastic Methods for Physics, Chemistry and Natural Sciences; Springer: Berlin/Heidelberg, Germany, 1990.

17. Peliti, L.; Vulpiani, A. (Eds.) Measures of Complexity; Springer: Berlin/Heidelberg, Germany, 1988.

18. Takayama, H. (Ed.) Cooperative Dynamics in Complex Systems; Springer: Berlin/Heidelberg, Germany, 1989. 
19. Carslaw, H.S.; Jaegher, J.C. Conduction of Heat in Solids; Clarendon Press: Oxford, UK, 1959.

20. Nelson, E. Dynamical Theories of Brownian Motion; Princeton University Press: Princeton, NJ, USA, 1967.

21. de Groot, S.R.; Mazur, P. Non-Equilibrium Thermodynamics; North-Holland: Amsterdam, The Netherlands, 1969.

22. Coley, W.T.; Kalmykov, Y.P.; Waldron, J.T. The Langevin Equation; World Scientific: Singapore, 1996. 